\title{
Fast quasi-periodic oscillations in the eclipsing polar VV Puppis from VLT and XMM-Newton observations
}

\author{
J. M. Bonnet-Bidaud ${ }^{1}$, M. Mouchet ${ }^{2}$, E. Falize ${ }^{3}$, L. Van Box Som ${ }^{3}$, C. Busschaert ${ }^{3}$, D. A. H. Buckley ${ }^{4}$, \\ H. Breytenbach ${ }^{4,5}$, T. R. Marsh ${ }^{6}$, R. P. Ashley ${ }^{6}$, and V. S. Dhillon ${ }^{7,8}$ \\ 1 Département d'Astrophysique-Laboratoire AIM, CEA/Irfu, 91191 Gif-sur-Yvette, France \\ e-mail: bonnetbidaud@cea.fr \\ 2 LUTH-Observatoire de Paris, UMR 8102-CNRS, Université Paris-Diderot, 92190 Meudon, France \\ 3 CEA-DAM-DIF, 91297 Arpajon, France \\ ${ }^{4}$ South African Astronomical Observatory, PO Box 9, Observatory 7935, Cape Town, South Africa \\ 5 Department of Astronomy, University of Cape Town, Rondebosch 7700, Cape Town, South Africa \\ ${ }^{6}$ Department of Physics, University of Warwick, Coventry CV4 7AL, UK \\ 7 Department of Physics and Astronomy, University of Sheffield, Sheffield S3 7RH, UK \\ ${ }^{8}$ Instituto de Astrofisica de Canarias, 38205 La Laguna, Tenerife, Spain
}

Received 19 April 2019 / Accepted 5 June 2019

\begin{abstract}
We present high time resolution optical photometric data of the polar VV Puppis obtained simultaneously in three filters $\left(u^{\prime}\right.$, HeII 14686, $r^{\prime}$ ) with the ULTRACAM camera mounted at the ESO-VLT telescope. An analysis of a long $50 \mathrm{ks}$ XMM-Newton observation of the source, retrieved from the database, is also provided. Quasi-periodic oscillations (QPOs) are clearly detected in the optical during the source bright phase intervals when the accreting pole is visible, confirming the association of the QPOs with the basis of the accretion column. QPOs are detected in the three filters at a mean frequency of $\sim 0.7 \mathrm{~Hz}$ with a similar amplitude $\sim 1 \%$. Mean orbitally-averaged power spectra during the bright phase show a rather broad excess with a quality factor $Q=v / \Delta v=5-7$ but smaller data segments commonly show a much higher coherency with $Q$ up to 30 . The X-ray Multi-mirror Mission XMM $(0.5-10 \mathrm{keV})$ observation provides the first accurate estimation of the hard X-ray component with a high $k T \sim 40 \mathrm{keV}$ temperature and confirms the high extreme ultraviolet (EUV)-soft/hard ratio in the range of 4-15 for VV Pup. The detailed X-ray orbital light curve displays a short $\Delta \phi \simeq 0.05$ ingress into self-eclipse of the active pole, indicative of an accretion shock height of $\sim 75 \mathrm{~km}$. No significant X-ray QPOs are detected with an amplitude upper limit of $\sim 30 \%$ in the range $0.1-5 \mathrm{~Hz}$. Detailed hydrodynamical numerical simulations of the post-shock accretion region with parameters consistent with VV Pup demonstrate that the expected frequencies from radiative instability are identical for X-rays and optical regime at values $v \sim 40-70 \mathrm{~Hz}$, more than one order magnitude higher than observed. This confirms previous statements suggesting that present instability models are unable to explain the full QPO characteristics within the parameters commonly known for polars.
\end{abstract}

Key words. accretion, accretion disks - novae, cataclysmic variables - white dwarfs - instabilities - X-rays: binaries pulsars: individual: VV Pup

\section{Introduction}

The star VV Pup was recognised very early on as a periodic variable star from the analysis of photographic plates taken at the now defunct Union Observatory (Johannesburg, South Africa). It was found to have a $100.4 \mathrm{~min}$ period, which was the shortest known period at the time (van Gent 1931). The binary nature of the source was later established from extended photometry and spectroscopy observations, leading to an accurate ephemeris (Walker 1965), which is still valid today, more than 50 years later. However, the true nature of the source was only later uncovered with the discovery of strong linear and circular polarisation (Tapia 1977), classifying VV Pup as a member of the AM Her stars, which are now more commonly known as magnetic cataclysmic variables (MCVs) or Polars.

MCVs are close interacting binaries consisting of a strongly magnetised ( $\gtrsim 10 \mathrm{MG}$ ) white dwarf (WD) accreting matter from a red dwarf companion via Roche lobe overflow (see reviews in Ferrario et al. 2015; Mukai 2017). The magnetic field is strong enough to capture matter near L1 and force the flow inside an accretion column down to the WD magnetic poles. This strong magnetic interaction usually leads to tidally locked systems in which the WD rotation period, which is fast originally, slows down to equate with the orbital period, leading to a stable geometry. Material falling down the column accelerates to a supersonic speed and a shock is formed above the WD surface, at the magnetic pole(s). Below the shock, the plasma cools by emitting bremsstrahlung in the X-rays and cyclotron radiation in the optical and infrared. These two cooling processes lead to stratification in both density and temperature. At the shock, the temperature reaches a few tens of $\mathrm{keV}$, depending mainly on the WD mass (see Cropper 1990; Warner 1995). According to the exact geometry of the system (e.g. orbital inclination and pole colatitude), mass transfer may feed one or two of the WD poles.

MCVs are rather "clean" systems in the sense that a unique, small emitting region, specifically the bottom of the accretion column below the shock and its immediate surroundings, largely dominate the output radiation. The emission nicely separates into different energy ranges, from infrared and optical (post-shock 
cyclotron emission), to ultraviolet and soft X-rays (heated WD surface) and to hard X-rays (post-shock thermal free-free emission).

Besides the energy distribution, other interesting diagnostics may come from the observed fast variability. Indeed, a sample of MCVs are known to show fast 1-3 s, quasi-periodic oscillations (QPOs) that were attributed to hydro-radiative instabilities in the post-shock zone, inducing shock height oscillations with a period of the order of the post-shock cooling time scale (Langer et al. 1981, 1982; Chevalier \& Imamura 1982). Fast QPOs were discovered by Middleditch in the early 1980s (Middleditch 1982) and currently, five MCVs (V834 Cen, AN UMa, EF Eri, BL Hyi and VV Pup) show 1-5 s QPOs with low-amplitude $1-5 \%$ in their optical emission. Coupling the shock energy distribution with the properties of the shock oscillations may theoretically provide a full determination of the major accretion parameters (WD mass, column cross section and accretion rate). Due to well-controlled physical processes, MCV accretion columns also represent a unique astrophysical environment that can be validly tested by both numerical simulations (Van Box Som et al. 2018a) and laser astrophysics experiments (Cross et al. 2016).

However, significant difficulties still exist to definitively attribute the observed QPOs to hydro-radiative instabilities. First of all, no X-ray QPOs have been detected in Polars so far, whereas high amplitude QPOs are predicted by shock models for an accretion column dominated by bremsstrahlung cooling. A systematic search in the XMM-Newton archive data (0.5$10 \mathrm{keV}$ ) for 20 of the brightest polars did not provide significant detection with upper limits constraining the physical parameters of the column (Bonnet-Bidaud et al. 2015, hereafter BB15). Also, 1D numerical hydrodynamic simulations for a large sample of polar physical parameters have shown that the dominant frequencies of the shock oscillations are generally an order of magnitude greater than the typical QPO detected frequencies (Busschaert et al. 2015; Van Box Som et al. 2018a). These inconsistencies show that the standard column model is not complete and that there is a lack of a fundamental physical ingredient. The reason why only a very limited number of MCVs (five out of more than one hundred) show these fast QPOs is also not clear.

Here, we report on new fast optical photometric observations of VV Pup, which were obtained at the VLT in order to better characterise the fast quasi-periodic oscillations and to compare their properties with what was expected from the standard accretion model and from improved magnetohydrodynamic (MHD) numerical simulations. A similar study was also conducted for the polar V834 Cen (Mouchet et al. 2017).

VV Pup is one MCV that has two identified poles with a surface field of $31.5 \mathrm{MG}$ and $54.6 \mathrm{MG}$. The magnetic field structure is well described by a $40 \mathrm{MG}$ dipole slightly offset from the WD centre (Wickramasinghe et al. 1989; Schwope \& Beuermann 1997; Mason et al. 2007). The binary system consists of a secondary star of mass of $0.10 \pm 0.02 M_{\odot}$ orbiting a $(0.73 \pm 0.05) M_{\odot} \mathrm{WD}$ (Howell et al. 2006). From data obtained by the Gaia mission, the VV Pup distance was recently and accurately measured to $137 \pm 1 \mathrm{pc}$ (Luri et al. 2018).

Only sparse and limited information on the source X-ray light curve and spectrum is available from previous observations (Patterson et al. 1984; Osborne et al. 1985; Ramsay et al. 1996; Imamura et al. 2000; Pandel \& Córdova 2005). Here, we also present the first detailed VV Pup X-ray light curve and spectrum during a high state, which was obtained from an extended XMM-Newton observation.

\section{Ultracam optical data}

\subsection{Observations and data reduction}

VV Pup was observed in May of 2005 during two nights (11 and 13 May 2005) at the ESO-VLT telescope using the ULTRACAM camera (Dhillon et al. 2007). This ultra-fast CCD camera was mounted as a visitor instrument on the UT3 Unit of the $8.2 \mathrm{~m}$ Very Large Telescope (VLT). It is a triple-beam device designed to provide high temporal photometry in three different filters simultaneously. The data were acquired using the Sloan Digital Sky Survey filters (SDSS) $u^{\prime}\left(\lambda_{\text {eff }}=3557 \AA\right)$ and $r^{\prime}$ $\left(\lambda_{\text {eff }}=6261 \AA\right)$ and a narrow-band filter centred close to the HeII line $\left(\lambda_{\text {eff }}=4662 \AA, 108 \AA\right.$ width $)$, indicated as He filter hereafter. This filter was not active during a portion of the first night.

A time resolution of $0.097 \mathrm{~s}$ was used to search for QPOs up to $5.1 \mathrm{~Hz}$ (Nyquist frequency). Data were obtained in a twowindow drift mode with a $4 \times 4$ binning. Weather conditions were good on 11 May 2005 (seeing 0.6"), but were less optimal on 13 May 2005 with a larger seeing (occurences up to $1.5^{\prime \prime}$ ) and the occasional presence of cirrus. The total exposure time was five hours $(18 \mathrm{ks})$. All orbital phases were covered at least three times. Table 1 gives a log of the observations.

The data were reduced using the ULTRACAM pipeline ${ }^{1}$. Corrections for bias and flat field response were applied. An airmass correction was also performed. To check for the sky and the instrument stability, differential photometry was performed using a nearby non-variable comparison. No absolute calibration was applied since we were focusing on the oscillation variability study.

\subsection{Orbital light curves}

Figure 1 shows the light curves for the three filters. The orbital phase is computed using the ephemeris given by Walker (1965), where phase zero refers to the maximum of the $\sim 1.67 \mathrm{~h}$ optical light curve. The separation for the two nights is shown by a vertical line marking a gap of 27 orbital cycles. The strong modulation of the light curves clearly indicates that VV Pup was in a high state during the observations. The main feature is a bright phase, $\phi \sim 0.75-1.15$, with a smooth rise and rapid drop observed similarly in the three wavelength bands. A very small bump at $\phi \sim 1.4$ is visible in the $r^{\prime}$ and $u^{\prime}$ bands during the first faint phase which might indicate a weak contribution from a second pole.

Our observations cover five bright phases (labelled B1 to B5) three of which are only partially covered (B2, B3, and B5). Strikingly, the maximum flux in B4 is significantly reduced, for instance, when compared to the B1 maximum by a factor of 5.8, 7.4 and 9.1 for the $r^{\prime}$, He and $u^{\prime}$ filters, respectively. Such variability from cycle to cycle was also observed in previously published light curves, though to a lesser extent (see for instance Fig. 1 in Imamura et al. 2000).

\subsection{QPO characteristics}

In order to search for QPOs and study their variability, we performed fast Fourier analysis of the light curves in the three filters for consecutive segments of 1024 points at $0.0975 \mathrm{~s}$ resolution (i.e. $99.8 \mathrm{~s}$ duration), using the powspec command in the

\footnotetext{
1 http://deneb.astro.warwick.ac.uk/phsaap/software/ ultracam/html/
} 
Table 1. VV Pup observing runs with ULTRACAM.

\begin{tabular}{llcrrrrrr}
\hline \hline Source & $\begin{array}{l}\text { Date } \\
\text { yy-mm-dd }\end{array}$ & $\begin{array}{c}\text { HJDstart } \\
2400000+\end{array}$ & $\begin{array}{c}\text { Exp. } \\
(\mathrm{s})\end{array}$ & $\begin{array}{c}\text { Res. } \\
(\mathrm{s})\end{array}$ & $\begin{array}{r}\text { Rate }\left(r^{\prime}\right) \\
\left(\mathrm{ct} \mathrm{s}^{-1}\right)\end{array}$ & Orb. phase & Obs. mode & Filters \\
\hline VV Pup & $05-05-11$ & 53502.47576389 & 1377 & 0.0975 & 14100 & $0.72-0.95$ & Drift & $u^{\prime} r^{\prime}$ \\
VV Pup & $05-05-11$ & 53502.49487269 & 5179 & 0.0975 & 16350 & $0.99-1.85$ & Drift & $u^{\prime}$, HeII, $r^{\prime}$ \\
VV Pup & $05-05-13$ & 53504.45811343 & 6525 & 0.0975 & 8414 & $0.14-1.22$ & Drift & $u^{\prime}$, HeII, $r^{\prime}$ \\
VV Pup & $05-05-14$ & 53504.53365741 & 4885 & 0.0975 & 11150 & $0.22-1.03$ & Drift & $u^{\prime}$, HeII, $r^{\prime}$ \\
\hline
\end{tabular}

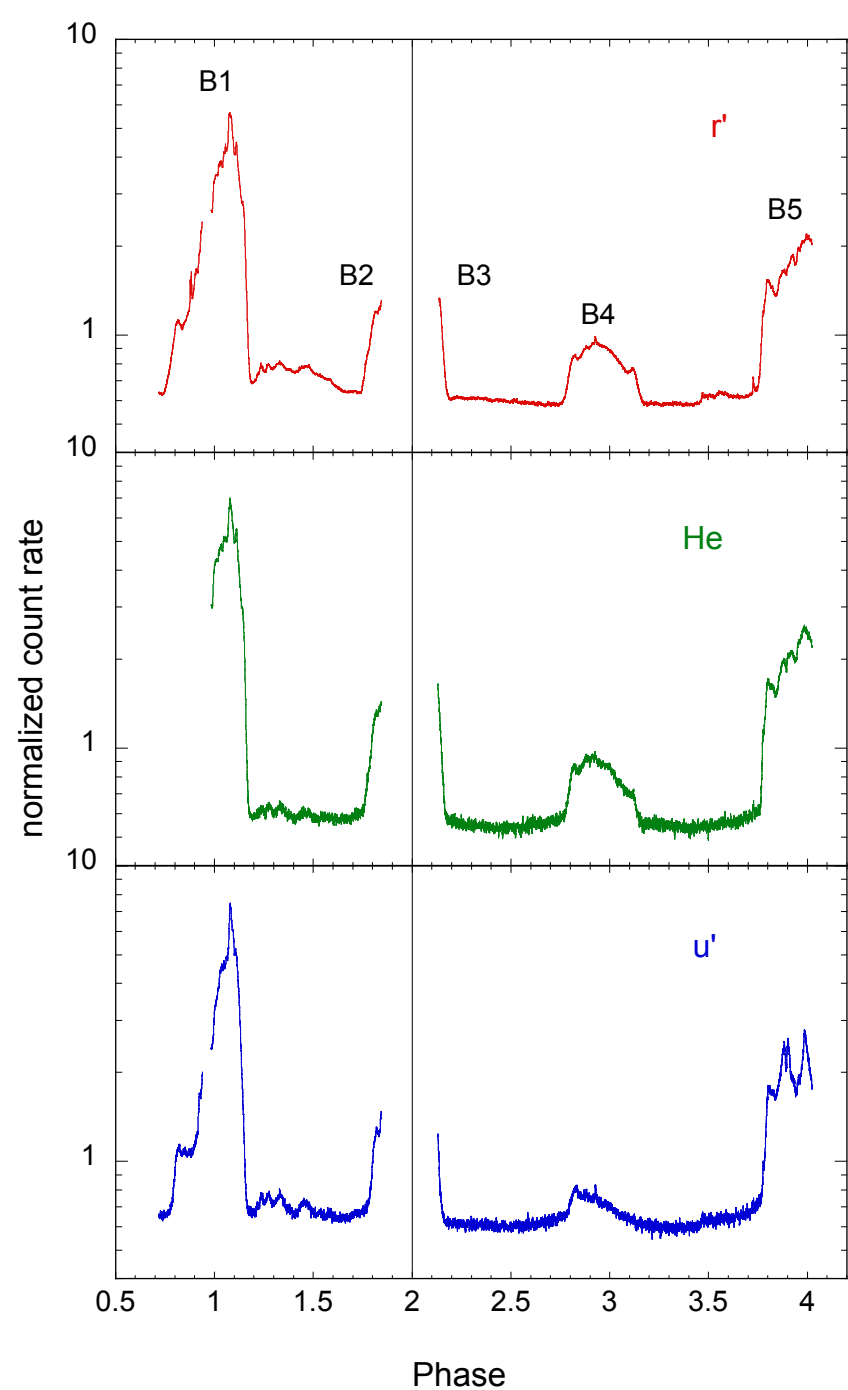

Fig. 1. Normalised light curves in the $r^{\prime}$ (top), He (middle) and $u^{\prime}$ (bottom) filters versus orbital phase at a resolution of $9.7 \mathrm{~s}$. The source light curve has been divided by the comparison one. Note that the vertical scale is logarithmic. The observations are spread over four different orbital cycles but split over two different nights (the vertical line indicates the gap of 27 orbital cycles). The different bright phase intervals (B1 to B5) are labelled.

HEASOFT Xronos package ${ }^{2}$. The significance level of the signal was estimated after its normalisation as defined by Leahy et al. (1983), such that the power distribution is a $\chi^{2}$ distribution with two degrees of freedom (d.o.f.). The $\chi^{2}$ distribution is additive; therefore, rebinning the Fast Fourier Transforms (FFTs) by first

\footnotetext{
2 https://heasarc.gsfc.nasa.gov/xanadu/xronos/xronos. html
}

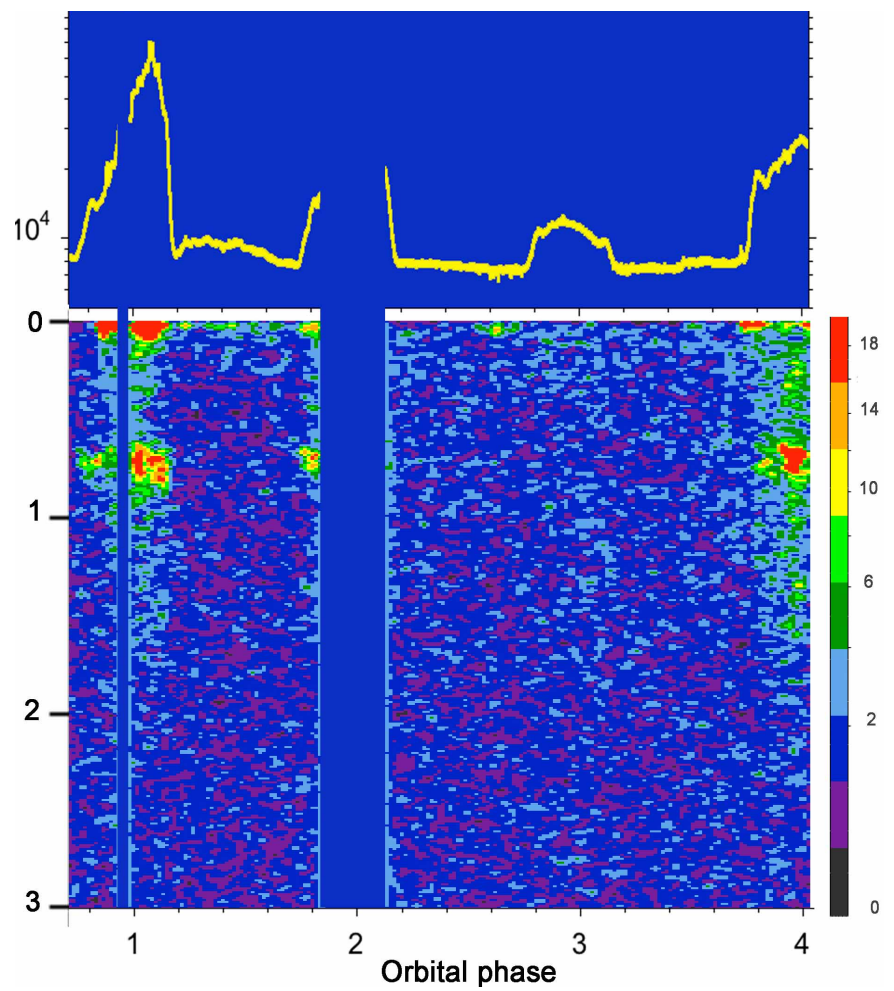

Fig. 2. 2D Time-frequency image of the power spectrum for the $r^{\prime}$ light curve. The $X$-axis is the orbital phase and the $Y$-axis is the frequency in the restricted range $0-3 \mathrm{~Hz}$. Colour-coded squared amplitude (with Leahy et al. 1983 normalisation) is shown according to the scale on the right. Individual (99.87 s) spectra are shown and the image has been smoothed with a 2-point Gauss filter. The corresponding $r^{\prime}$ light curve is shown at the top.

averaging $\mathrm{W}$ frequency bins and then averaging the $\mathrm{M}$ individual FFTs results in a statistical distribution of power following a $\chi^{2}$ distribution with $2 \mathrm{WM}$ d.o.f. For $M=1$ and $W=1$, the $99 \%$ confidence detection level corresponds to a power value of 21.7 (see van der Klis et al. 1988).

Figure 2 displays a 2D image of the power spectrum versus orbital phase in the range of $0-3 \mathrm{~Hz}$. QPOs around $\sim 0.7 \mathrm{~Hz}$ are only clearly detected during the bright phases. However, there is an exception during the anomalous B4 bright phase, which is significant.

Mean optical QPO amplitudes were computed by averaging FFTs from data in the bright phase $(\phi=0.75-1.15)$ and faint phase $(\phi=0.15-0.75)$ intervals and the results are given in Table 2. For the bright phase, amplitudes were evaluated by first subtracting a power-law fitted outside the $0.5-1 \mathrm{~Hz}$ range and then computing the mean root-mean-square (rms) values in the $0.5-1 \mathrm{~Hz}$ interval. Error bars are evaluated from the effect 
Table 2. Mean optical $0.5-1 \mathrm{~Hz}$ QPO properties.

\begin{tabular}{|c|c|c|c|}
\hline Filter (Rate ct s ${ }^{-1}$ ) & $\operatorname{rms}(\%)$ & $\begin{array}{l}f_{\text {med }} \\
(\mathrm{Hz})\end{array}$ & $\begin{array}{c}F W H M \\
(\mathrm{~Hz})\end{array}$ \\
\hline \multicolumn{4}{|c|}{ Bright $\phi=(0.75-1.15)^{(*)}$} \\
\hline$r^{\prime}(24402)$ & $1.04 \pm 0.06$ & $0.73 \pm 0.01$ & $0.11 \pm 0.01$ \\
\hline $\mathrm{He}(3228)$ & $1.27 \pm 0.70$ & $0.73 \pm 0.01$ & $0.14 \pm 0.02$ \\
\hline$u^{\prime}(4542)$ & $0.97 \pm 0.80$ & $0.75 \pm 0.01$ & $0.14 \pm 0.02$ \\
\hline \multicolumn{4}{|c|}{ Faint $\phi=(0.15-0.75)$} \\
\hline$r^{\prime}(8239)$ & $<0.15 \pm 0.36$ & - & - \\
\hline $\mathrm{He}(981)$ & $<0.57 \pm 0.96$ & - & - \\
\hline$u^{\prime}(1486)$ & $<0.52 \pm 0.92$ & - & - \\
\hline
\end{tabular}

Notes. ${ }^{(*)}$ Excluding B4.

of adding or subtracting to the power a standard deviation computed in the range $3-5 \mathrm{~Hz}$. The mid-frequency is given as $f_{\text {med }}$, the median value in the range $0.5-1 \mathrm{~Hz}$. The measured mean amplitudes are of the order of $\sim 1 \%$ of the total brightness in the three filters and their frequencies are very similar $(\sim 0.74 \mathrm{~Hz})$. The QPO widths (FWHM) are of the order of $0.11 \mathrm{~Hz}\left(r^{\prime}\right)$ and up to $0.14 \mathrm{~Hz}$ (He and $u^{\prime}$ ). It is interesting to note that the bright phase, B4, was excluded as no significant QPOs are detected here.

For the faint phase, the power in the $0.5-1 \mathrm{~Hz}$ range was computed after subtracting a second order polynomial continuum fitted outside $0.5-1 \mathrm{~Hz}$. Also, the error bars were computed just as for the bright phase. The upper limits are given in Table 2 with all amplitudes consistent with zero and best constrained to $<0.15 \%$ in the $r^{\prime}$ filter, which confirms the absence of QPOs during this phase.

Figure 3 shows the QPO frequency profiles averaged during all bright phases (excluding B4); the characteristics are given in Table 2 . Besides the excess around $\sim 0.74 \mathrm{~Hz}$, there is a hint of additional power around $\sim 1.5 \mathrm{~Hz}$ in the three filters; it is the most pronounced in $u^{\prime}$ though with significant noise variability.

We checked the variability in the different orbital cycles by building a mean FFT for the different bright phases (B1 to B5), shown in Fig. 4 for the $r^{\prime}$ filter. QPOs are detected well in all bright phases, even in the short B3 interval. However, they are absent in the bright phase, B4. The shape of the QPOs is variable, showing clear multiple components in B2 and B5. The rms amplitudes for the four intervals, B1, B2, B3, and B5 are of $0.86 \%, 1.34 \%, 0.97 \%$, and $1.39 \%$, respectively. Also, the upper limit for B4 is $<0.40 \%$.

\subsection{QPOs orbital and time variability}

We also computed mean FFTs in 20 phase bins across the orbit to look for QPO orbital modulation. The measured QPO rms amplitudes are shown in Fig. 5 (lower panel) and the corresponding $r^{\prime}$ mean counting rate is also shown (upper panel). There is no strict correlation between the relative amplitude and the flux, the QPOs are present at nearly the same relative amplitude $(\sim 1-$ $1.5 \%$ ) during the entire bright phase.

Since the individual quasi-periodic pulsations are seen by the naked eye, we also investigated the QPO characteristics down to short time intervals ( $\sim 50 \mathrm{~s})$. As an example, Fig. 6 shows one of the intervals where the QPOs are most prominent with a typical mean amplitude $\sim 4 \%$ at a mean period of $\sim 1.47 \mathrm{~s}$. Inspecting other intervals clearly reveals that the oscillating signal significantly varies on a timescale of minutes in the same way as already observed in the source V834 Cen (Mouchet et al. 2017).

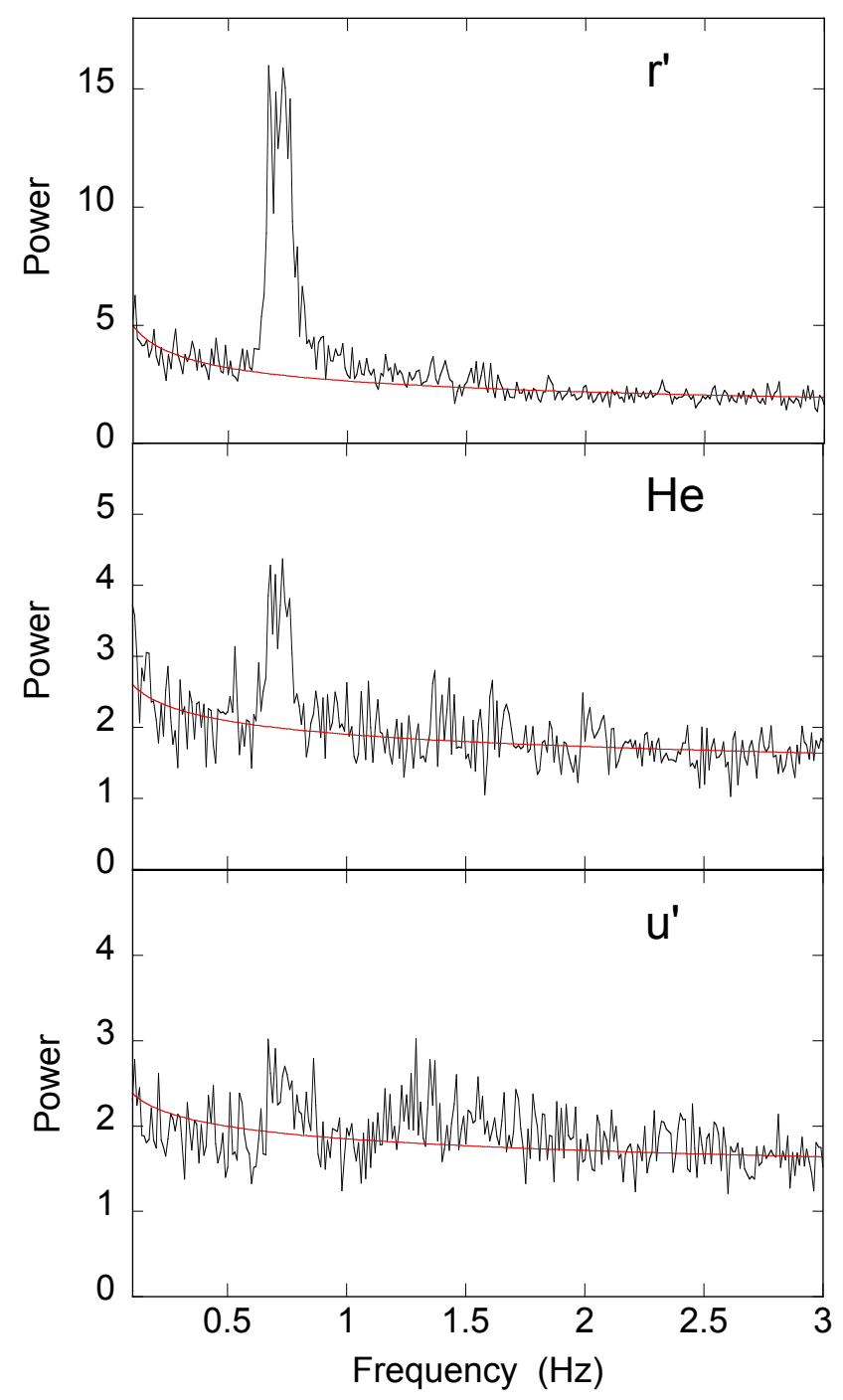

Fig. 3. Bright phase QPO frequency profiles. Mean power density spectra are shown in the range of $0.1-3 \mathrm{~Hz}$ for the bright phase interval $0.75-$ 1.15 , excluding B4, in the three wavelength bands (top: $r^{\prime}$, middle: He, bottom: $\left.u^{\prime}\right)$. The $Y$-scale is the power computed with Leahy normalisation. The red line shows the subtracted background fitted as a power-law outside the $0.5-1 \mathrm{~Hz}$ interval.

\section{XMM-Newton X-ray observations}

VV Pup was observed with the XMM-Newton satellite for a long duration $\sim 50 \mathrm{ks}$ exposure on 20 October 2007 . The observation in timing mode covers eight consecutive orbital cycles of the source during a high state and has not been fully published yet. In the context of the analysis of a set of selected polars performed by Bonnet-Bidaud et al. (2015), an upper limit on fast X-ray QPOs in VV Pup in the range of $0.1-5 \mathrm{~Hz}$ was set at $\sim 28 \%$ during the bright phase of this observation in 2007. Previous observations have provided only limited information on the source light curve and spectrum based on Einstein, EXOSAT, ROSAT and RXTE satellites (Patterson et al. 1984; Osborne et al. 1985; Ramsay et al. 1996; Imamura et al. 2000). An XMM observation of VV Pup is available, but it is restricted to a typical low state of the source (Pandel \& Córdova 2005). XMM data were retrieved so as to provide a useful comparison for our optical data. For our purposes, we restrict the study to the EPIC-pn data, obtained in timing mode 


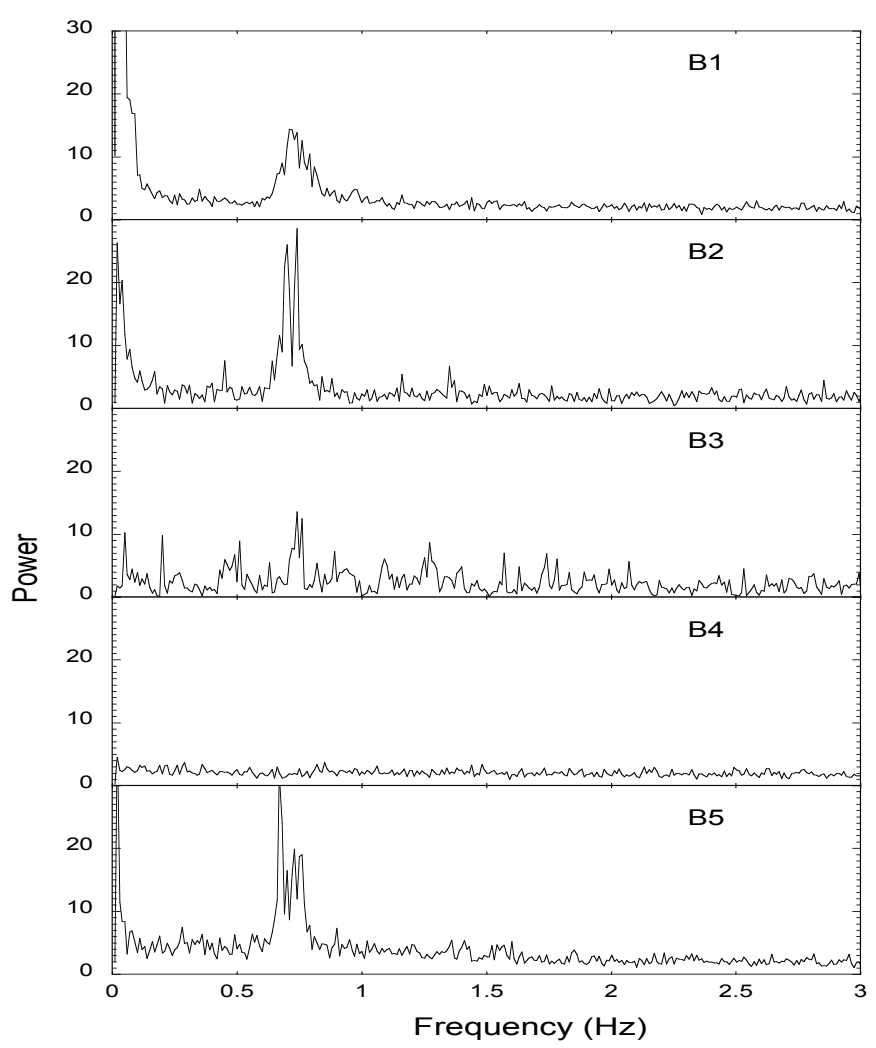

Fig. 4. Power spectra for the $r^{\prime}$ light curve in the different bright phases as labelled in Fig. 1. We note the disappearance of the QPOs in B4.

(Strüder et al. 2001). The resulting count rate $\left(<10 \mathrm{cts} \mathrm{s}^{-1}\right)$ is well below the pile-up limit. Since a high energy $(>10 \mathrm{keV})$ background flaring is not apparent, the total observation was kept. The data were processed using the version V11.0.0 of the Science Analysis Software (SAS) and FTOOLS software packages ${ }^{3}$. We included both single and double events (PATTERN $\leqq 4)$ in our analysis. The barycentric correction was applied for the timing analysis. The source spectrum was extracted by selecting a 15column strip around RAWX $=37$, corresponding to the brightest signal in the image. The background spectrum was extracted in columns 4-18, far away from the source.

\subsection{X-ray light curves}

The total XMM observation covers eight consecutive orbital cycles. Light curves were produced in the ranges of $0.5-2 \mathrm{keV}$ (soft), $2-12 \mathrm{keV}$ (hard), and $0.5-12 \mathrm{keV}$ (full) with resulting mean net count rates of $0.24,0.11$, and $0.35 \mathrm{ct} \mathrm{s}^{-1}$, respectively. Figure 7 shows the mean X-ray orbital light curves in the three bands, which are folded with the $0.0697 \mathrm{~d}$ orbital period. The mid X-ray maximum occurs at phase $0.953 \pm 0.003$ (median determination) with respect to the optical maximum as predicted by Walker's ephemeris (Walker 1965), indicating a very stable orbital and spin period as already noted by Howell et al. (2006). The shape of the X-ray bright phase is slightly asymmetrical with a progressive rise and a steeper slope during fall. For comparison, the optical light curve $\left(r^{\prime}\right)$ corresponding to the B1 cycle (Fig. 1) is also shown superposed. The optical asymmetry is more pronounced and the

\footnotetext{
3 https://www. cosmos.esa.int/web/xmm-newton/ how-to-use-sas
}

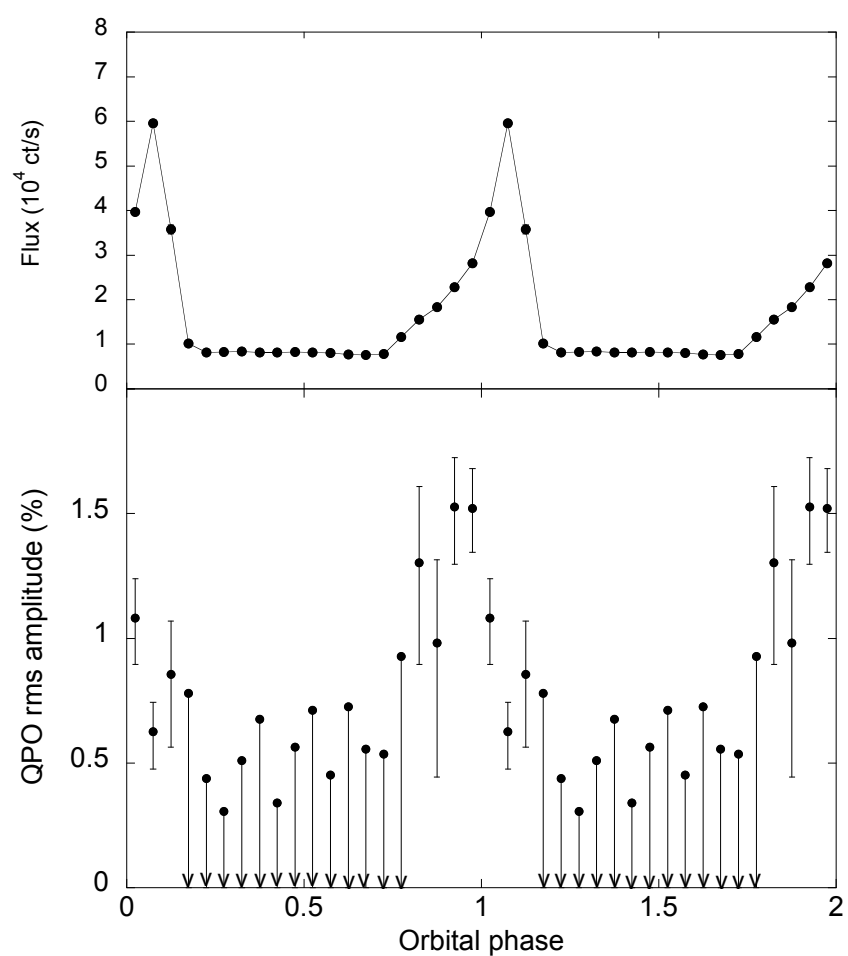

Fig. 5. Orbital variation of the QPO rms amplitudes in 0.05 phase intervals for the $r^{\prime}$ filter (bottom). Only upper limits are derived in the faint phase interval $(\phi=0.15-0.75)$. The corresponding $r^{\prime}$ folded light curve is also shown (top).

mid-maximum is at phase $1.047 \pm 0.005$. This significant phase shift was commonly observed revealing that the optical bright phase is variable in position and width (Warner \& Nather 1972; Schwope \& Beuermann 1997; Imamura et al. 2000). Although the period is reliable over several decades, fluctuations of the accretion flow most probably induce small variations in the location of the accreting region.

\subsection{X-ray $Q P O$ search}

In BB15, X-ray QPOs were already searched in the frequency range up to $50 \mathrm{~Hz}$ in the $0.5-10 \mathrm{keV}$ energy range. Here, we extend the search in the frequency range up to $125 \mathrm{~Hz}$ by using a EPIC-pn time resolution of $0.004 \mathrm{~s}$ and analyse the data in the three separate energy ranges as defined above. We applied the same procedure as in BB15 to derive upper limits on X-ray QPO amplitudes, according to van der Klis et al. (1988).

Averaged power spectra, normalised according to Leahy et al. (1983), are computed adding individual FFTs of 1024 points intervals. In none of the average spectra did the maximum power exceed the detection limit, $P_{\text {detect }}$ where $P_{\text {detect }}$ is the power level that has only a $1 \%$ probability to be exceeded by the noise. The upper limit is then obtained as $P_{\mathrm{UL}}=P_{\max }-P_{\text {exceed }}$, where $P_{\max }$ is the highest power in the selected frequency range, and $P_{\text {exceed }}$ is the power level which will be exceeded by only $1 \%$ of the data, in the absence of any periodic signal due to noise. Results are given in Table 3 in the three energy ranges. The relative amplitudes were computed in two frequency ranges $0.1-5 \mathrm{~Hz}$ and $5-125 \mathrm{~Hz}$, assuming a standard frequency of $1 \mathrm{~Hz}$ and $20 \mathrm{~Hz}$, respectively. The upper limits for the relative QPO amplitudes are high $(\sim 30-50 \%)$, which are in agreement with values published in BB15. Individual power spectra in short 

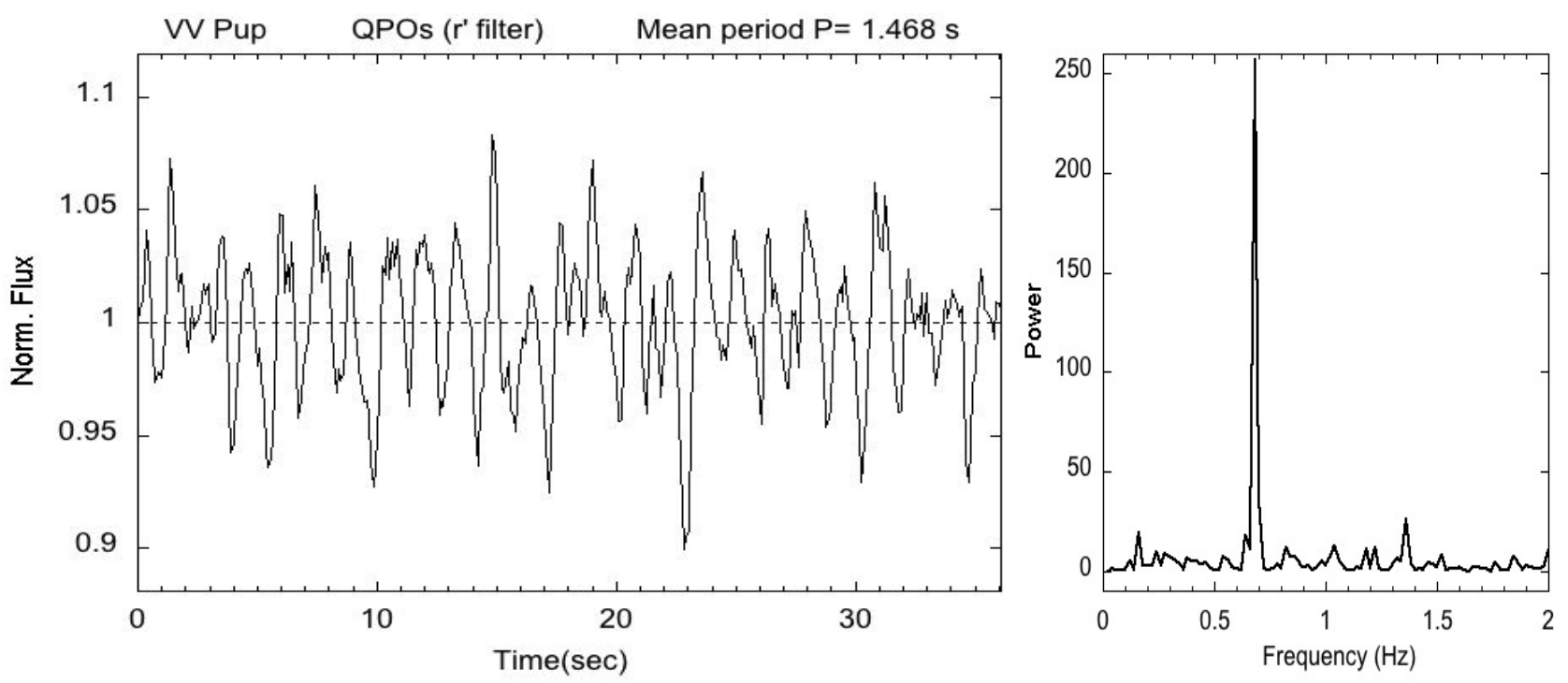

Fig. 6. Example of typical VV Pup flux oscillations observed in a $36 \mathrm{~s}$ time interval at mid-phase 0.970 (left). The shape and amplitude of the oscillations strongly vary with a mean amplitude of $\sim 4 \%$ in a narrow frequency range of $\sim 0.68 \mathrm{~Hz}$ as shown by the power spectrum (right).

Table 3. X-ray fast oscillation $0.1-5$ and $5-125 \mathrm{~Hz}$ detection limits from XMM-EPIC-pn observations.

\begin{tabular}{lllllllll}
\hline \hline Energy range & Time resolution & $\begin{array}{l}\text { Rate } \\
\left(\mathrm{ct} \mathrm{s}^{-1}\right)\end{array}$ & $M$ & $\begin{array}{l}f_{\max } \\
(\mathrm{Hz})\end{array}$ & $P_{\max }$ & $\begin{array}{l}P_{\text {exceed }} \\
2.6 \sigma\end{array}$ & $\begin{array}{l}P_{\text {detect }} \\
2.6 \sigma\end{array}$ & $\begin{array}{l}\text { Limit } \\
\% \mathrm{rms}\end{array}$ \\
\hline $0.5-12 \mathrm{keV}$ & $0.1 \mathrm{~s}$ & 0.76 & 191 & 3.42 & 2.56 & 1.68 & 2.65 & 29.6 \\
$0.5-2 \mathrm{keV}$ & $0.1 \mathrm{~s}$ & 0.50 & 191 & 2.60 & 2.38 & 1.68 & 2.65 & 29.8 \\
$2-12 \mathrm{keV}$ & $0.1 \mathrm{~s}$ & 0.26 & 191 & 0.61 & 2.56 & 1.68 & 2.65 & 41.7 \\
$0.5-12 \mathrm{keV}$ & $0.004 \mathrm{~s}$ & 0.76 & 4582 & 40.53 & 2.08 & 1.93 & 2.12 & 51.3 \\
$0.5-2 \mathrm{keV}$ & $0.004 \mathrm{~s}$ & 0.50 & 4582 & 27.10 & 2.06 & 1.93 & 2.12 & 58.9 \\
$2-12 \mathrm{keV}$ & $0.004 \mathrm{~s}$ & 0.26 & 4582 & 12.45 & 2.06 & 1.93 & 2.12 & 78.5 \\
\hline
\end{tabular}

104 s intervals were also examined since QPOs may be transient; however, positive signals were not detected.

\subsection{X-ray energy spectrum}

The VV Pup X-ray energy spectrum was generated using the associated redistribution matrix, ancillary response files, and the standard SAS threads. Spectral fits were performed using the XSPEC v12.7 HEASOFT software package ${ }^{4}$, in the 0.5$10 \mathrm{keV}$ range where the calibration accuracy and the count rate statistics are the best. In this section, all errors are given at a $90 \%$ confidence level for one interesting parameter $\left(\Delta \chi^{2}=2.71\right)$.

The source spectrum was accumulated for the bright phase, corresponding to the orbital phase interval $(0.75-1.15)$. We first fitted the data with the simplest X-ray spectrum for polars, consisting of the sum of a blackbody and a bremsstrahlung component, with absorption by cold matter (wabs model). The neutral column density was fixed at $3 \times 10^{19}$ at $\mathrm{cm}^{-2}$, the best fit value derived from EUVE observations (Vennes et al. 1995). An Fe line with a Gaussian profile at $6.76 \mathrm{keV}$ was also required in the fit. The results are given in Table 4 . The data are best fitted with a $0.14 \mathrm{keV}$ blackbody and a high temperature of $39 \mathrm{keV}$ bremsstrahlung, indicating a high WD mass. We checked the influence of the $N_{\mathrm{H}}$ value by increasing it by a factor of ten. The value is still lower than that of the total interstellar one in

\footnotetext{
4 https://heasarc.gsfc.nasa.gov/xanadu/xspec
}

the direction of the source. This did not change the blackbody temperature but the bremsstrahlung temperature is slightly lower (33 keV).

A partial covering with neutral absorption (pcfabs model) was also tested, which led to a lower bremsstrahlung temperature $(13 \mathrm{keV})$, a small covering fraction of 0.27 and a neutral absorption column of $1.0 \times 10^{23} \mathrm{at} \mathrm{cm}^{-2}$. However the fit was not significantly improved (reduced $\chi^{2}=1.270$ for 133 d.o.f.). A reflection of the X-ray continuum onto the WD surface can harden the underlying incident spectrum. Adding a convolution of the bremsstrahlung with a reflect component (Magdziarz \& Zdziarski 1995) does indeed decrease the bremsstrahlung temperature $(18 \mathrm{keV})$. However it does not significantly improve the fit $\left(\chi^{2}=1.264\right.$ for 134 d.o.f. $)$ and it requires a unrealistic, large reflection factor of 3.0.

A more physical approach is described by a stratified post shock region (PSR; Suleimanov et al. 2005, 2016). The model ipolar was initially developed for intermediate polars, by specifying the capture point of the material by the magnetic field lines in terms of the WD radius. Here we chose to fix the capture radius at $30 R_{\mathrm{WD}}$ (Meggitt \& Wickramasinghe 1989), which is consistent with a capture inside the Roche lobe of the WD (models are nearly indistinguishable for capture radii greater than $30 R_{\mathrm{WD}}$ ). The results of this fit are given in Table 4 and shown in Fig. 8. The best fit gives a high mass compatible with the maximum of $1.4 M_{\odot}$. This is consistent with the high bremsstrahlung temperature found above. The blackbody component and the Gaussian line are also quite similar 


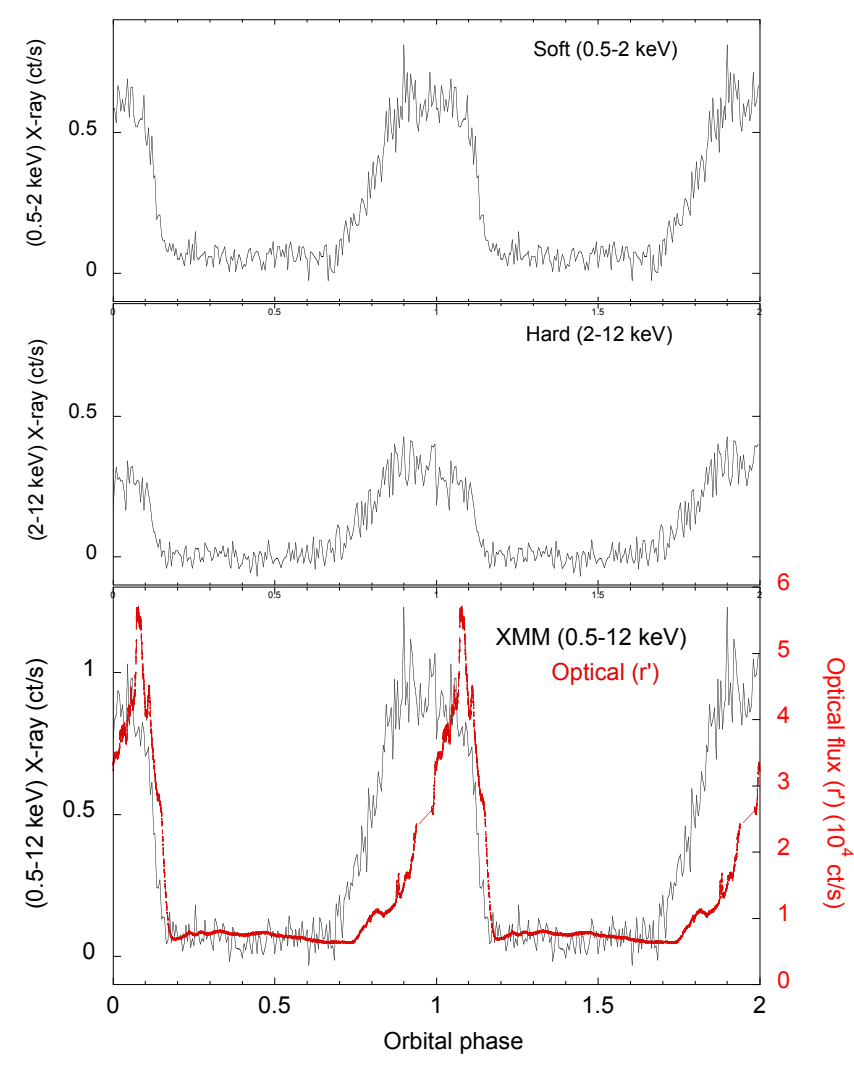

Fig. 7. Mean X-ray orbital light curve of VV Pup in the two different bands: soft (0.5-2 keV) (top), hard (2-12 keV) (middle), and in the full range $(0.5-12 \mathrm{keV})($ bottom $)$. The X-ray light curve is an average of eight consecutive cycles. For comparison, the optical light curve $\left(r^{\prime}\right)$, corresponding to B1, is also shown superposed (in red).

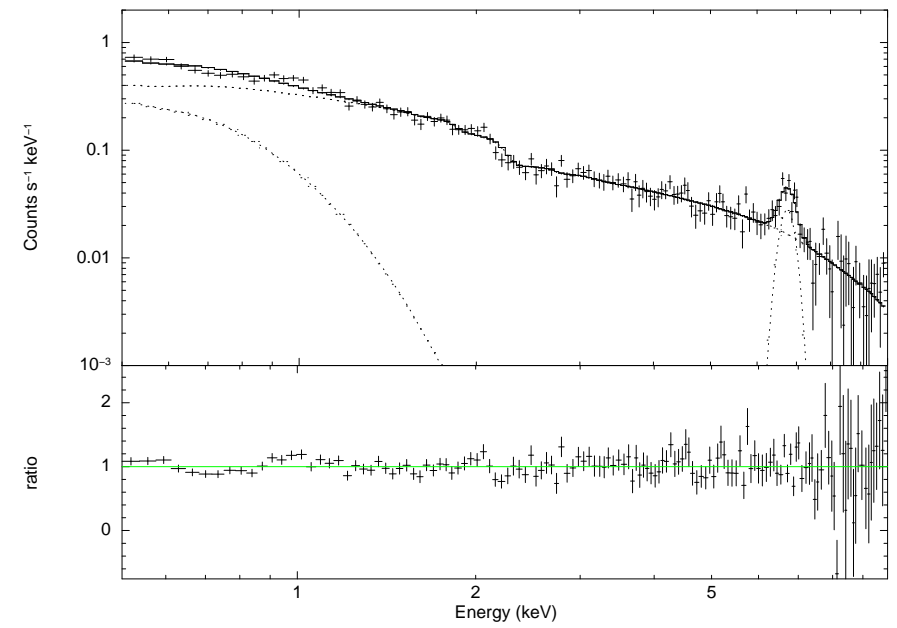

Fig. 8. Bright phase X-ray spectrum fitted with the ipolar model which parameters are given in Table 4 (upper frame) and data/model ratio (lower frame). The dotted lines represent the separate component contributions.

to the blackbody-bremsstrahlung fit. If a neutral partial covering is added to the fit, the WD mass decreases to $0.59 M_{\odot}$ (with $\chi_{\text {red }}^{2}=1.255$ for 133 d.o.f.). However, this value is not well constrained by the fit with a range of $0.35-1.38 M_{\odot}$ for a $1-\sigma$ uncertainty.

The results from the different models seem to indicate the presence of a hard component, pointing to a high temperature bremsstrahlung or similarly, a high WD mass. How- ever, the restricted XMM energy range is not well suited to definitively evaluate such a hard component, which is better constrained by data above $15 \mathrm{keV}$. We find that the use of more sophisticated models (neutral partial covering, and reflection) gives either lower values for the temperature of the bremsstrahlung or for the WD mass in the ipolar model. Nevertheless, the fit is not significantly improved. Unfortunately, the VV Pup WD mass is still poorly constrained from X-ray data. A RXTE observation also did not provide useful constraint at high energy since the source was rather faint and only detectable up to $5 \mathrm{keV}$ (Imamura et al. 2000).

Using the precise Gaia distance of $137 \pm 1 \mathrm{pc}$ (Luri et al. 2018, DR2, Gaia catalogue), the normalisation coefficients can be used to constrain the accretion geometry. Assuming typical values of densities and column cross-section as $n_{\mathrm{e}}=n_{\mathrm{i}}=$ $10^{16} \mathrm{~cm}^{-3}$ and $S=4 \times 10^{14} \mathrm{~cm}^{2}$ (see below), the normalisation of the bremsstrahlung component is consistent with a shock height of $h_{\mathrm{s}}=56 \mathrm{~km}$. The normalisation of the minor blackbody component leads to a very small emitting surface (fraction of the WD surface of $2.4 \times 10^{-8}$ for a $0.73 M_{\odot}$ ), The blackbody/bremsstrahlung ratio is $\sim 0.05$ in the $0.5-10 \mathrm{keV}$ range and the total unabsorbed $0.5-10 \mathrm{keV}$ luminosity is $7.4 \times 10^{30} \mathrm{erg} \mathrm{s}^{-1}$.

\section{Discussion}

VV Pup is the archetype of two-pole polars that only shows one active pole most of the time. The parameters of the binary system, with $i=75^{\circ}$, indicate that the primary southern pole with $B=31 \mathrm{MG}$ is at a colatitude $\beta=145-155^{\circ}$ and self-eclipsed during a significant part of the orbit (Liebert \& Stockman 1979; Wickramasinghe et al. 1989; Schwope \& Beuermann 1997). The WD mass is reasonably well constrained to $0.73 \pm 0.05 M_{\odot}$ from the secondary orbital velocity measured from detailed infrared spectroscopy during low-state (Howell et al. 2006). Also this value will be used hereafter. Even though the existence of VV Pup has been known for a long time, it was the third source classified as a polar, (Tapia 1977), detailed information has not yet been published on its X-ray light curve and spectrum for energies up to $10 \mathrm{keV}$. Previous results from earlier satellites were mostly confined to below $5 \mathrm{keV}$ from the Einstein satellite (Patterson et al. 1984), EXOSAT (Osborne et al. 1985; Mason 1985), ROSAT (Ramsay et al. 1996) and RXTE (Imamura et al. 2000).

\subsection{The soft $X$-ray problem}

Early VV Pup X-ray data from a restricted energy range $(0.15-$ $4.0 \mathrm{keV}$ ) had pointed to a suspected high soft/hard ratio of $\sim 15$, leading to the concept of a "soft X-ray machine" (Patterson et al. 1984). The present XMM data provide the first precise estimate for the hard component. Our best fit indicates a high temperature $\sim 39 \mathrm{keV}$ (bremsstrahlung model) or a massive WD (ipolar model). We note that the best fitted mass value, close to the maximum Chandrasekhar mass, is probably an overestimate due to the limited XMM energy range. Hard components, $k T>15 \mathrm{keV}$, are indeed only weakly constrained in the $0.5-10 \mathrm{keV}$ range. An additional small soft $0.14 \mathrm{keV} \mathrm{X}$-ray excess is also required in the fit but contributes negligibly to the total flux. The total observed $0.5-10 \mathrm{keV}$ flux is $3.27 \times 10^{-12} \mathrm{erg} \mathrm{cm}^{-2} \mathrm{~s}^{-1}$, corresponding to a luminosity of $L_{\mathrm{X}}$ of $7.4 \times 10^{30} \mathrm{erg} \mathrm{s}^{-1}$ and the total unabsorbed bolometric luminosity is $2.0 \times 10^{31} \mathrm{erg} \mathrm{s}^{-1}$.

From EUV $(0.069-0.18 \mathrm{keV})$ and soft X-ray (0.1$2 \mathrm{keV}$ ) observations, a major soft component was detected (Patterson et al. 1984; Ramsay et al. 1996; Vennes et al. 1995). The exact temperature is not clear and is in the range of 
Table 4. Spectral fits of XMM-EPIC-pn data.

\begin{tabular}{llllll}
\hline \hline \multicolumn{3}{c}{ Model bb + brems + Gaussian } & \multicolumn{3}{c}{ Model bb + ipolar + Gaussian } \\
\hline Model component & Parameters & Value & Model component & Parameters & Value \\
\hline BB & $k T_{\text {bb }}(\mathrm{keV})$ & $0.142_{-0.018}^{+0.017}$ & BB & $k T_{\text {bb }(\mathrm{keV})}$ & $0.138_{-0.016}^{+0.021}$ \\
& Norm. $\left(10^{-6}\right)$ & $3.68_{-0.45}^{+0.50}$ & & Norm. $\left(10^{-6}\right)$ & $3.55_{-0.47}^{+0.69}$ \\
BREMS & $k T_{\text {br }}(\mathrm{keV})$ & $39.3_{-13.5}^{+33.2}$ & IPOL & $M_{\mathrm{WD}}\left(M_{\odot}\right)$ & $1.40_{-0.09}^{\text {pegged }}$ \\
& Norm. $\left(10^{-4}\right)$ & $5.65_{-0.39}^{+0.77}$ & & Norm. $\left(10^{-30}\right)$ & $2.66_{-0.072}^{+9.14}$ \\
GAUSS & E (keV) & $6.760_{-0.046}^{+0.047}$ & GAUSS & E (keV) & $6.760_{-0.046}^{+0.047}$ \\
& $\sigma(\mathrm{keV})$ & $0.179_{-0.047}^{+0.053}$ & & $\sigma(\mathrm{keV})$ & $0.179_{-0.045}^{+0.052}$ \\
Flux $(0.5-10 \mathrm{keV})$ & $\left(\mathrm{erg} \mathrm{s}^{-1} \mathrm{~cm}^{-2}\right)$ & $3.27 \times 10^{-12}$ & Flux $(0.5-10 \mathrm{keV})$ & $\left(\mathrm{erg} \mathrm{s} \mathrm{cm}^{-2}\right)$ & $3.16 \times 10^{-12}$ \\
$L_{\mathrm{X}}(0.5-10 \mathrm{keV})$ & $\left(\mathrm{erg} \mathrm{s}^{-1}\right)$ & $7.4 \times 10^{30}$ & $L_{\mathrm{X}}(0.5-10 \mathrm{keV})$ & $\left.(\mathrm{erg} \mathrm{s})^{-1}\right)$ & $7.12 \times 10^{30}$ \\
& $\chi_{\text {red }}^{2}($ d.o.f. $)$ & $1.261(135)$ & & $\chi_{\text {red }}^{2}(\mathrm{~d} .0 . f)$. & $1.258(135)$ \\
\hline
\end{tabular}

$10-40 \mathrm{eV}$ with a best fitted value of $k T \sim 26 \mathrm{eV}$ from EUVE data. Furthermore, absorption is also poorly constrained and in the range of (1.9-3.7) $\times 10^{19} \mathrm{~cm}^{-2}$ (Vennes et al. 1995). Assuming a blackbody distribution with $k T=26 \mathrm{eV}$ and $N_{\mathrm{H}}=3 \times 10^{19} \mathrm{~cm}^{-2}$, the EUV flux corresponds to a soft bolometric luminosity of $8.6 \times 10^{31} \mathrm{erg} \mathrm{s}^{-1}$ and $3.2 \times 10^{32} \mathrm{erg} \mathrm{s}^{-1}$ respectively with and without absorption.

The EUV-soft over XMM-hard ratio is therefore in the range of $\sim 4-15$, confirming the major contribution at low energy. We stress, however, that the EUV-soft emission is still poorly constrained due to uncertainties in the temperature and absorption that have a major impact on the estimated luminosity. The soft $\mathrm{X}$-ray excess in polars is thought to arise from the irradiation of the polar cap and dense blobs accreted within the WD photosphere (Warner 1995).

\subsection{VV Pup: An eclipsing system}

VV Pup exhibits simple X-ray and optical light curves, consisting of a bright phase covering nearly half of the orbital cycle during which the optical and X-ray emission of the lower part of the accretion column above the pole is visible. In addition, a faint phase corresponds to an interval when this emission region is eclipsed by the body of the white dwarf. The observation of strong linear polarisation at the end of the bright phase corroborates this picture (Cropper \& Warner 1986; Piirola et al. 1990).

The detailed XMM light curve shows a bright phase of duration of $\Delta \phi=0.46 \pm 0.02$ with an asymmetric shape characterised by a slow rise of $\Delta \phi=0.14 \pm 0.01$ and an abrupt decline of $\Delta \phi=0.05 \pm 0.01$. There is no significant change between the soft $(0.5-2 \mathrm{keV})$ and hard $(2-10 \mathrm{keV})$ light curves. This shape is similarly present in the EUVE range (Vennes et al. 1995). The optical light curve is slightly different. In particular, it has a much more gradual egress $(\Delta \phi \sim 0.25)$ but a similar very sharp ingress. This is comparable to what has previously been observed, though the optical pole egress is also seen to be shorter in duration in some cases and sometimes more similar to X-rays (Imamura et al. 1993, 2000). As seen in Fig. 7, we note the small shift in phase between the X-ray and optical observations obtained at different dates. Some jitter was already noted by previous authors, indicating small changes in the pole location. However, the ephemeris is remarkably stable on long timescale.
The sharp ingress $\Delta \phi=0.05 \pm 0.01$ can be used to constrain the size of the emitting region. The ingress into self-eclipse corresponds to the interval between the time the column is perpendicular to our field of view and the time when the top of the column disappears behind the line of sight. According to Larsson (1989), the ratio $h / R_{\mathrm{WD}}$, where $h$ is the shock height delimiting the cyclotron emission region and $R_{\mathrm{WD}}$ the WD radius, can be computed from the ingress duration $\Delta \phi$ and source parameters $\left(i=75^{\circ}, \beta=150^{\circ}\right)$ as $h / R_{\mathrm{WD}} \sim 0.01$. For a WD mass of $0.73 M_{\odot}$, this corresponds to $h \sim 76 \mathrm{~km}$.

The hard X-ray flux attributed to the optically thin bremsstrahlung emission of the post-shock region is geometrically modulated by the self eclipse. From a detailed numerical simulation of the post-shock region, X-ray emission can be modelled and typical X-ray light curves can be computed according to the source accretion parameters (see Sect. 4.4). Figure 9 shows the predicted $0.5-10 \mathrm{keV}$ light curve for a variable inclination and representative source geometrical parameters (colatitude $\beta=150^{\circ}$ and column cross-section $S=4 \times 10^{14} \mathrm{~cm}^{2}$ ). The visible part of the accretion column during an orbital phase is evaluated by pure-geometrical effects according to the source parameters (WD parameters, column radius, column height and the $i$ and $\beta$ angles). The simulated $\mathrm{X}$-ray light curves are derived by integrating the simulated $\mathrm{X}$-ray luminosity through the visible part. We note that a perfect circular cross-section is assumed for the column here. The general shape is well reproduced, in particular the sharp ingress, for a value $i$ close to $75^{\circ}$ but not the egress-ingress asymmetry. The more gradual egress may in fact be indicative of a deviation from pure circular shape, pointing to a possible elongated polar spot. In the same way, the optical flux associated with the optically thick cyclotron emission will also be affected by different projection and limb-darkening effects. We note that the very similar shape of the optical curves in the different filters, with equivalent egress and ingress times, excludes a pure absorption effect here.

\subsection{The fast optical oscillations}

VV Pup was the fourth polar found to exhibit optical $\sim 1 \mathrm{~s}$ QPOs. This feature is not a transient feature and is present during all high states of the source for which observations at high temporal resolution were performed (Larsson 1989; Imamura et al. 1993, 2000) and is confirmed with our ULTRACAM observations. 


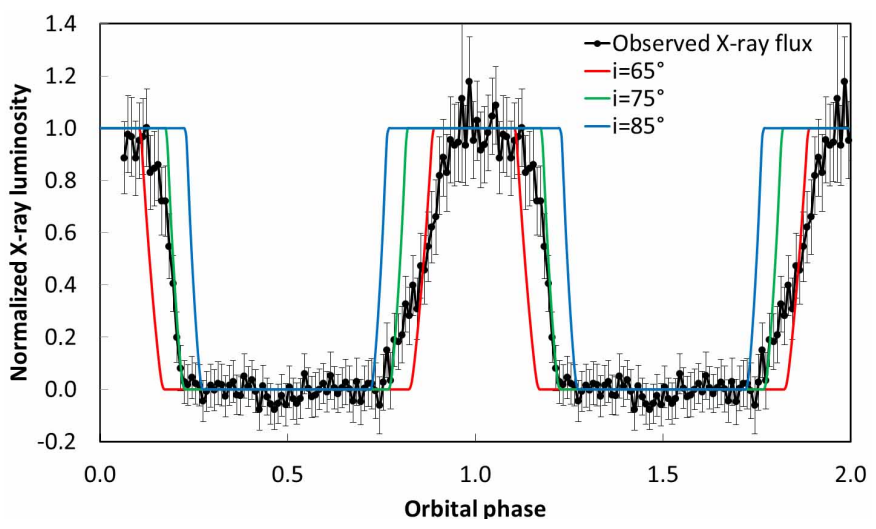

Fig. 9. Simulated X-ray light curves according to the source parameters (see text), for different system inclinations (red $i=65^{\circ}$, green $75^{\circ}$, and blue $85^{\circ}$ ), compared to the observed XMM light curve (in black).

VV Pup being a pole-eclipse system offers a simple way to directly link the QPOs to the accretion region above the pole. Our observations clearly show that optical QPOs are only observed during the restricted bright interval $(\phi=0.75-1.15)$ when the pole region is seen and not in the faint part $(\phi=0.15-$ 0.75), confirming previous results (Larsson 1989; Imamura et al. 1993). The QPOs non-detection during faint phases confirms their link with the cyclotron emission which forms the bulk of the optical bright phase radiation. It is interesting to note, however, that the upper limit in faint phase $(<0.2-0.5 \%)$ does not preclude the possibility of lower amplitude QPOs that may come from the second pole.

For the first time, our observations also clearly show that the QPOs may disappear during a full orbital cycle (B4) when the maximum flux is significantly reduced. As shown in Fig. 4, QPOs are however detected in the preceding and following cycles. This behaviour is not fully explained. In B4, the maximum flux compared to B1 is reduced by a factor of approximatively six and nine for the red $\left(r^{\prime}\right)$ and blue $\left(u^{\prime}\right)$ filters, respectively. Similar flux variations from cycle to cycle are also apparent in previous observations but with a more reduced factor of approximatively two (see Fig. 1 in Imamura et al. 2000). One possible explanation might be a temporary lower mass transfer linked to small changes in the capture region, close to the inner Lagrangian point. In this case, the disappearance of the QPOs show that they are strongly dependent on the accretion rate.

The optical QPOs are detected with comparable amplitude $\sim 1 \%$ at the same frequencies of $\sim 0.7 \mathrm{~Hz}(1.4 \mathrm{~s})$ in the three filters from red to blue and also including the narrow filter HeII (4662 ̊). This band was primarily selected to constrain the QPO amplitude in the emission lines (see Mouchet et al. 2017). However, the underlying continuum is still the dominant fraction of the flux in the filter. The measurement uncertainty prevents an accurate measurement of the contribution of the line flux to the QPOs. However, the fact that the QPO amplitude in this filter is similar to the other bands might indicate that the line flux also contributes to the QPOs.

The orbital variability (see Fig. 5) shows that QPOs are clearly detected from the beginning of the polar cap egress with approximatively a constant $\sim(1-1.5 \%)$ relative amplitude during the entire bright phase until the sharp decrease of the ingress. This is in accordance with the visibility of the polar cap and accretion column.
The true nature of the QPOs is not yet clear. They may be the consequence of a naturally broad-band process, in which different frequencies are excited simultaneously, or the result of a narrow-band feature varying in frequency over time. Obviously, averaging QPOs over long time intervals will always result in a broad feature, hiding the true QPO nature. The mean frequency profile, shown in Fig. 3 with values given in Table 2, shows that the typical FWHM is $\Delta v=(0.11-0.14) \mathrm{Hz}$. For a damped oscillator, this would correspond to a mean quality factor $Q=v / \Delta v=5-7$. However, for the typical $40 \mathrm{~s}$ interval as shown in Fig. 6, the quality factor reaches up to $Q=31$. In this case, the coherence time $\tau$, defined as $\tau=2 Q / v$, is $\sim 90 \mathrm{~s}$, under the assumption of a sine wave shot with an exponentially decaying amplitude with a time constant. Such values are higher than what was previously reported for VV Pup by Imamura et al. (1993) However, it is interesting to note their different definition of coherence. These values are also similar to what is observed in V834 Cen (Mouchet et al. 2017). The amplitudes of the highcoherency intervals are also much higher, reaching more than $5 \%$. It is therefore more likely that the QPOs are some kind of superposition of these narrow-band features.

\subsection{Hydrodynamical simulations}

The standard interpretation of the fast QPOs process is in terms of hydro-radiative instability in the post-shock accretion region. Their frequencies are indeed characteristic of the timescale of shock oscillations driven by the cooling instability (see the review by $\mathrm{Wu} 2000)$. Using the multidimensional hydrodynamic code RAMSES (Teyssier 2002), the radiation hydrodynamics equations can be solved to simulate the radiative accretion dynamics in the accretion column. The radiative losses in the accretion column are modelled by a cooling function, including both the optically thin bremsstrahlung, and the optically thick cyclotron approximated by a power-law (see Busschaert et al. 2015; Van Box Som et al. 2018a for details).

From such numerical simulations, relevant synthetic light curves and X-ray spectra can be extracted, depending on four basic source parameters (WD mass, magnetic field B, net accretion rate $\dot{M}$, and accretion cross-section $S$ ). The code was run for a set of parameters relevant to VV Pup with $M_{\mathrm{WD}}=0.73 M_{\odot}, B=31 \mathrm{MG}$ and a total accretion rate of $\dot{M}=0.25 \times 10^{16} \mathrm{~g} \mathrm{~s}^{-1}$, which was derived assuming that the total luminosity (EUV + soft + hard X-ray) of $L_{X}=3.4 \times 10^{32} \mathrm{erg} \mathrm{s}^{-1}$ corresponds to the gravitational energy of the accreted matter.

No direct measure of the column cross-section $S$ is available. Only rough estimations can be derived from both spectral and geometrical considerations on the soft X-ray emission, which can be attributed to the heated hot spot at the basis of the column. From extreme ultraviolet (EUV) and far-ultraviolet (FUV) observations, different estimations are given, ranging from $4 \times$ $10^{14} \mathrm{~cm}^{2}$ (Vennes et al. 1995) to $5 \times 10^{15} \mathrm{~cm}^{2}$ (Hoard et al. 2002), respectively. The size deduced from UV observations is likely to be an overestimate, since UV emission encompasses cooler and larger regions than the limited basis of the accretion column.

However, complementary constraints can come from the numerical simulations, if other parameters are fixed, such as the column height. For example, in a given set of source parameters, varying only the column cross-section results in a monotonic increase of the shock height. For typical VV Pup parameters, a cross-section varying from $2 \times 10^{14}$ to $10^{15} \mathrm{~cm}^{2}$ results in an increase of the shock height from 50 to $100 \mathrm{~km}$. Thereby, 


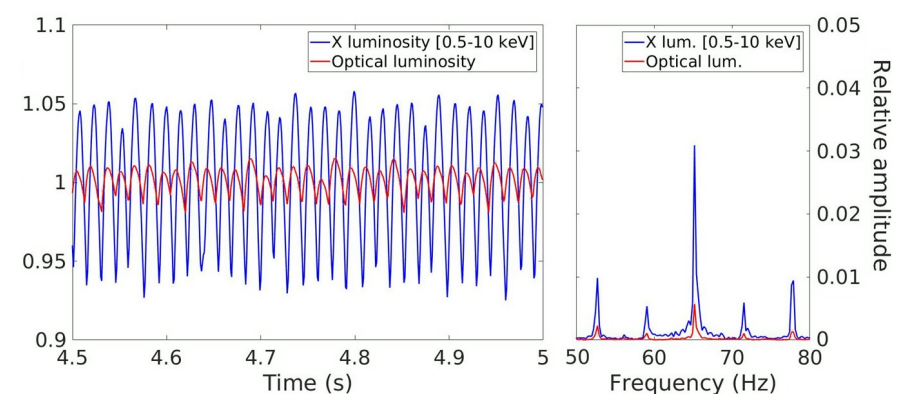

Fig. 10. Luminosity oscillations in X-rays (in blue) and optical (in red) predicted by 1D-numerical RAMSES simulations for the VV Pup parameters $\left(M_{\mathrm{WD}}=0.73 M_{\odot}, B=31 \mathrm{MG}, S=4 \times 10^{14} \mathrm{~cm}^{2}\right.$ section and $\dot{M}=2.5 \times 10^{15} \mathrm{~g} \mathrm{~s}^{-1}$ ). The corresponding FFTs are shown on the right with identical central frequencies at $\sim 65 \mathrm{~Hz}$ and X-ray and optical amplitudes of $6.8 \%$ and $1.6 \%$ respectively.

assuming a height of $h \sim 75 \mathrm{~km}$, as derived from the eclipse, indicates a cross-section close to $S \sim 4 \times 10^{14} \mathrm{~cm}^{2}$ as the best value.

Figure 10 shows the predicted flux oscillations in X-rays (bremsstrahlung) and optical (cyclotron) for a $4 \times 10^{14} \mathrm{~cm}^{2}$ section and a total accretion rate of $2.5 \times 10^{15} \mathrm{~g} \mathrm{~s}^{-1}$. Oscillations are present in X-rays and optical with the same dominant frequency of $65.2 \mathrm{~Hz}$ and with amplitudes of $6.8 \%$ and $1.6 \%$ respectively. When compared to observations, our upper limits on X-ray oscillations of $\sim 30 \%$ (in the $0.1-5 \mathrm{~Hz}$ range) and $\sim 50 \%$ (in the $5-125 \mathrm{~Hz}$ range) are not significantly constraining. Also in the optical, the present resolution does not allow us to search for predicted frequencies over $5 \mathrm{~Hz}$. However, the optical frequencies detected at $0.6-0.8 \mathrm{~Hz}$ are clearly not predicted by the numerical simulations, as shown in Figs. 6 and 10. Therefore, the observations obviously do not confirm the results of the numerical simulations. The same discrepancy was already noted for the source V834 Cen (Mouchet et al. 2017).

An extensive study using results from simulations covering a wide-range of polar parameters demonstrates that lower than $\sim 1 \mathrm{~Hz}$ QPO frequencies require a combination of parameters inconsistent with the observed ones (Van Box Som et al. 2018a). Namely, for a given white dwarf mass, magnetic field and accretion rate, an increase of the cross-section will have the effect of increasing the cyclotron radiative losses and lowering the frequencies of the oscillations. But at the same time, the amplitudes are also severely reduced to below the detected level, due to the strong damping effect of the cyclotron emission, so that no oscillation will be observed.

This is also in accordance with similar 1D simulations that solve the system dynamical evolution, using the MHD code PLUTO (Bera \& Bhattacharya 2018). In this study, numerical results computed for accretion parameters, and adapted to the polar V834 Cen, show that an increase in the column crosssection (in their case a pole radius $r_{\mathrm{p}}>200 \mathrm{~km}$ ) is required to stay in the observed optical frequency range. But, as a corollary, a negligible oscillation amplitude $\ll 0.1 \%$ is produced. In this respect, we would like to point out that their conclusions are somewhat misleading since, contrary to what stated in their abstract, the characteristics of the post-shock region are not consistent with the observed properties in these simulations.

We note also that some discrepancies exist in their predicted frequencies compared to our recent simulations (Van Box Som et al. 2018a). These differences are due to different assumptions. First, for the cyclotron/bremsstrahlung cooling ratio (noted $\epsilon_{\mathrm{s}}$ ), we use the more recent and more rigorous definition given by Saxton (1999), which is more adapted when the WD gravitation is taken into account. Bera \& Bhattacharya (2018) used a cooling function dependent on the column height (Langer et al. 1982), which is not appropriate for these numerical simulations. Also, as the time-dynamical QPOs are not self-similar, the oscillation characteristics depend on the initial conditions. Consequently, considering the steady-state solutions as initial conditions, as done in Bera \& Bhattacharya (2018), introduces a bias. In our simulations, the initial conditions are set by a homogeneous accretion flow striking the WD surface and creating an accretion shock. A non self-similar secondary shock developing near the WD photosphere then starts the oscillations (see Van Box Som et al. 2018a). The results are therefore non strictly comparable, but we do consider that our approach is more rigorous. However, some caution should be taken into account since the interpretation of the present simulations does not yet include detailed boundary conditions, such as the possible influence of the soft X-ray emitting polar cap on the incoming accretion flow.

\section{Conclusions}

VV Pup is an interesting polar as it is a pole-eclipse system that shows optical QPOs, allowing more detailed diagnostics:

- Optical QPOs are clearly detected here with approximatively the same flux fraction across the visible spectrum, during observations obtained over two different days. However, the observations show, for the first time, a remarkable interval when QPOs stopped for a full orbital period, during a significant optical flux variation.

- The QPOs are always solely observed during the bright phase, indicating that the QPO region is eclipsed and therefore likely linked to the bottom of the accretion column.

- Though the overall mean QPOs appear broadly spread over a $0.6-0.9 \mathrm{~Hz}$ interval, there are repetitive time intervals when the QPOs are nearly coherent for durations up to a few minutes, suggesting that the QPO frequency distribution most likely results from a superposition of discrete frequencies.

- Our analysis provides a first estimate of the X-ray spectrum up to $10 \mathrm{keV}$ that indicates a somewhat harder spectrum than expected from the WD mass, though this result requires further observations at higher energies. No X-ray QPOs are detected but the observed upper limit is still too high to put useful constraints on the models.

The observations are still generally in line with what is expected from the post-shock region above the WD magnetic pole, the main emitting region. However, there are still major discrepancies to be fully in accordance with the model. For the consistent parameters, detailed $1 \mathrm{D}$-simulations failed to jointly reproduce the observed frequencies and amplitudes. As there are no clear alternatives to the shock model, this may indicate major limitations in the treatment of the boundary conditions above and below the shock region and possibly more complex 2D/3D effects (Busschaert et al. 2015; Van Box Som et al. 2018a). As the simulations commonly show the presence of oscillations for a rather wide range of parameters, another intriguing point is also the small fraction of polars showing QPOs. This may possibly indicate the need of fine tuning between the accretion parameters $(\dot{M}, S)$. The sudden disappearance of the QPOs observed here, from one orbital cycle to the other, may be an example of conditions in which this fine tuning is suppressed due to possible changes in the geometry of the capture region with associated variation in the accretion rate. 
Further studies are clearly needed before the oscillations observed in polars can be used to provide powerful diagnostics of the WD accretion columns. These studies would be in the form of 2D and 3D numerical simulations and possibly laser astrophysics experiments. Indeed, we have demonstrated that the new powerful lasers devices, such as LMJ (Laser MegaJoule, France) and NIF (National Ignition Facility, USA), allow us to reproduce a scaled model of accretion shock (Falize et al. 2011). The ability to concentrate around $500 \mathrm{~kJ}$ of laser energy in millimetric volume of matter during a few nanoseconds leads to the production of a small-scale accretion column in a similar regime (Van Box Som et al. 2018b). The dynamics, the density, and temperature profiles typical of the post-shock region can be achieved and measured with the various diagnostics developed at these facilities (see review in Remington et al. 2006). Preliminary promising results have already been obtained with lasers of intermediate energy (Cross et al. 2016) and current designs are under way for experiments with megajoule lasers (Van Box Som et al., in prep.).

Acknowledgements. Part of this work was supported by the French Programme National de Physique Stellaire (PNPS) of the CNRS/INSU co-funded by CEA and CNES. DAHB's research is supported by the National Research Foundation of South Africa.

\section{References}

Bera, P., \& Bhattacharya, D. 2018, MNRAS, 474, 1629

Bonnet-Bidaud, J. M., Mouchet, M., Busschaert, C., Falize, E., \& Michaut, C. 2015, A\&A, 579, A24

Busschaert, C., Falize, É., Michaut, C., Bonnet-Bidaud, J.-M., \& Mouchet, M. 2015, A\&A, 579, A25

Chevalier, R. A., \& Imamura, J. N. 1982, ApJ, 261, 543

Cropper, M. 1990, Space Sci. Rev., 54, 195

Cropper, M., \& Warner, B. 1986, MNRAS, 220, 633

Cross, J. E., Gregori, G., Foster, J. M., et al. 2016, Nat. Commun., 7, 11899

Dhillon, V. S., Marsh, T. R., Stevenson, M. J., et al. 2007, MNRAS, 378, 825

Falize, É., Michaut, C., \& Bouquet, S. 2011, ApJ, 730, 96

Ferrario, L., de Martino, D., \& Gänsicke, B. T. 2015, Space Sci. Rev., 191, 111

Hoard, D. W., Szkody, P., Ishioka, R., et al. 2002, AJ, 124, 2238
Howell, S. B., Harrison, T. E., Campbell, R. K., Cordova, F. A., \& Szkody, P. 2006, AJ, 131, 2216

Imamura, J. N., Middleditch, J., Scargle, J. D., et al. 1993, ApJ, 419, 793

Imamura, J. N., Steiman-Cameron, T. Y., \& Wolff, M. T. 2000, PASP, 112, 18

Langer, S. H., Chanmugam, G., \& Shaviv, G. 1981, ApJ, 245, L23

Langer, S. H., Chanmugam, C., \& Shaviv, G. 1982, ApJ, 258, 289

Larsson, S. 1989, A\&A, 217, 146

Leahy, D. A., Darbro, W., Elsner, R. F., et al. 1983, ApJ, 266, 160

Liebert, J., \& Stockman, H. S. 1979, ApJ, 229, 652

Luri, X., Brown, A. G. A., Sarro, L. M., et al. 2018, A\&A, 616, A9

Magdziarz, P., \& Zdziarski, A. A. 1995, MNRAS, 273, 837

Mason, K. O. 1985, Space Sci. Rev., 40, 99

Mason, E., Wickramasinghe, D., Howell, S. B., \& Szkody, P. 2007, A\&A, 467, 277

Meggitt, S. M. A., \& Wickramasinghe, D. T. 1989, MNRAS, 236, 31

Mouchet, M., Bonnet-Bidaud, J.-M., Van Box Som, L., et al. 2017, A\&A, 600, A53

Mukai, K. 2017, PASP, 129, 062001

Osborne, J., Mason, K. O., Bonnet-Bidaud, J. M., Beuermann, K., \& Rosen, S. 1985, in X-ray Astronomy '84, eds. M. Oda, \& R. Giacconi, 63

Pandel, D., \& Córdova, F. A. 2005, ApJ, 620, 416

Patterson, J., Beuermann, K., Lamb, D. W., et al. 1984, ApJ, 279, 785

Piirola, V., Coyne, G. V., \& Reiz, A. 1990, A\&A, 235, 245

Ramsay, G., Cropper, M., \& Mason, K. O. 1996, MNRAS, 278, 285

Remington, B. A., Drake, R. P., \& Ryutov, D. D. 2006, Rev. Mod. Phys., 78, 755

Saxton, C. J. 1999, PhD Thesis, Univ. Sydney, Australia

Schwope, A. D., \& Beuermann, K. 1997, Astron. Nachr., 318, 111

Strüder, L., Briel, U., Dennerl, K., et al. 2001, A\&A, 365, L18

Suleimanov, V., Revnivtsev, M., \& Ritter, H. 2005, A\&A, 443, 291

Suleimanov, V., Doroshenko, V., Ducci, L., Zhukov, G. V., \& Werner, K. 2016, A\&A, 591, A35

Tapia, S. 1977, IAU Circ., 3054, 1

Teyssier, R. 2002, A\&A, 385, 337

Van Box Som, L., Falize, É., Bonnet-Bidaud, J.-M., et al. 2018a, MNRAS, 473, 3158

Van Box Som, L., Falize, E., Koenig, M., et al. 2018b, High Power Laser Sci. Eng., 6, E35

van der Klis, M. 1988, in Timing Neutron Stars, eds. H. Ogelman, \& E. P. J. van den Heuvel (Dordrecht: Kluwer), NATO ASI Series C, 262, 27 van Gent, H. 1931, Bull. Astron. Inst. Neth., 6, 93

Vennes, S., Szkody, P., Sion, E. M., \& Long, K. S. 1995, ApJ, 445, 921

Walker, M. F. 1965, Commmun. Konkoly Obs. Hung., 57, 1

Warner, B. 1995, Camb. Astrophys. Ser., 28

Warner, B., \& Nather, R. E. 1972, MNRAS, 156, 305

Wickramasinghe, D. T., Ferrario, L., \& Bailey, J. 1989, ApJ, 342, L35

Wu, K. 2000, Space Sci. Rev., 93, 611 